\title{
Copper-Catalyzed Asymmetric Umpolung Hydroamination: an Unified Strategy for the Synthesis of Chiral $\beta$-Amino Acid and Its Derivatives
}

Ge Zhang, ${ }^{\text {a† }}$ Yujie Liang, ${ }^{\text {b† }}$ Tao Qin, ${ }^{\mathrm{a}}$ Tao Xiong, ${ }^{* \mathrm{a}}$ Shuyu Liu, ${ }^{\mathrm{a}}$ Wei Guan, ${ }^{* \mathrm{~b}}$ and Qian Zhang*ac

\begin{abstract}
Catalytic asymmetric aza-Michael represents one of the most convenient and atomeconomical approach for the rapid construction of biologically active chiral $\beta$-amino acid frameworks. However, the direct enantioselective addition of nitrogen-based nucleophiles to intrinsically low reactivity of $\alpha, \beta$-unsaturated carboxylic acid, ester, and amide, as well as simple $\alpha, \beta$-unsaturated nitrile, remains a long-standing challenge. Herein, we reported an unified $\mathrm{Cu}$ catalyzed asymmetric umpolung hydroamination, which is capable of direct preparation of a series of $\beta$-amino acid, ester, amide, and nitrile in a highly regio-and enantioselective manner, without the requirement of traditional pre-installation of stoichiometric quantities of auxiliaries.
\end{abstract}

Catalytic asymmetric 1,4-addition of nitrogen-based nucleophiles to the Michael acceptors, namely aza-Michael addition reaction, has attracted continuous attention in recent decades, ${ }^{1}$ owing to furnishing one of the most convenient and atom-economical approach for the rapid construction of biologically and synthetically extremely important chiral $\beta$-amino acid frameworks from readily accessible feedstocks. ${ }^{2}$ In this regard, impressive advances have been made for the chemo- and enantioselective addition of nitrogen-based nucleophiles to highly reactive Michael acceptors, ${ }^{1}$ such as $\alpha, \beta$-unsaturated aldehydes, ketones, nitroolefins and vinyl sulfones. However, the corresponding addition to intrinsically less reactive $\alpha, \beta$-unsaturated acid, ester, and amide has far less been well established. ${ }^{1,3}$ Inspired by enzymatic systems activated unreactive carboxylic acids, the preponderant strategy up to now typically relys on the pre-installation of an activating functional group on the carbonyl to enhance the reactivity and increase enantioselectiivty (Scheme $1 \mathrm{~A}$, top). ${ }^{4}$ Nevertheless, the requirement of pre-installation and subsequent deprotection of stoichiometric quantities of activating auxiliaries are time-consuming multistep sequences, and sometimes might lead to chiral products racemization and/or incompatible with delicate molecular architectures when removal of these auxiliaries. To our knowledge, only some sporadic examples described the direct addition to $\alpha, \beta$-unsaturated acid $^{5}$ and $\alpha, \beta$-unsaturated ester $^{6}$ as well as enzyme-catalyzed transformations so far. ${ }^{7}$ Furthermore, the simple $\alpha, \beta$-unsaturated nitriles $(\beta$-substituted acrylonitrile), another typical kind of Michael acceptor and versatile building blocks in synthetic chemistry, ${ }^{8}$ the enantioselective addition with nitrogen-based nucleophiles to form a chiral center at the $\beta$-position of the nitrile group is only up to $22 \%$ enantiomeric excess (ee) value (Scheme $1 \mathrm{~A}$, 
bottom). ${ }^{9}$ More importantly, up to now, there remains no an unifiled satrategy which is sutiable for all of these challenging Michael acceptors. Therefore, exploitation of a general and alternative approach which is capable of rapid preparation of chiral $\beta$-amino acid derivatives from unmasked $\alpha, \beta$-unsaturated acid, ester, amide, even for simple $\alpha, \beta$-unsaturated nitrile, is high demand.

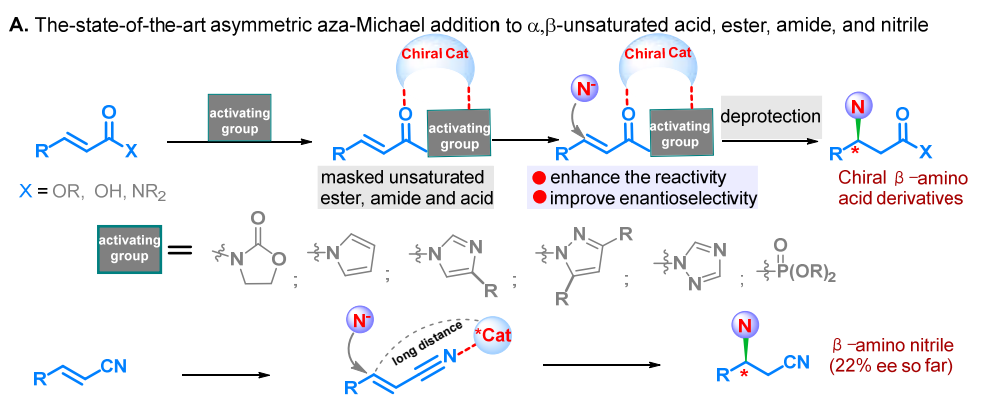

B. Previous reports on CuH-catalysed asymmetric transformations of Michael acceptors

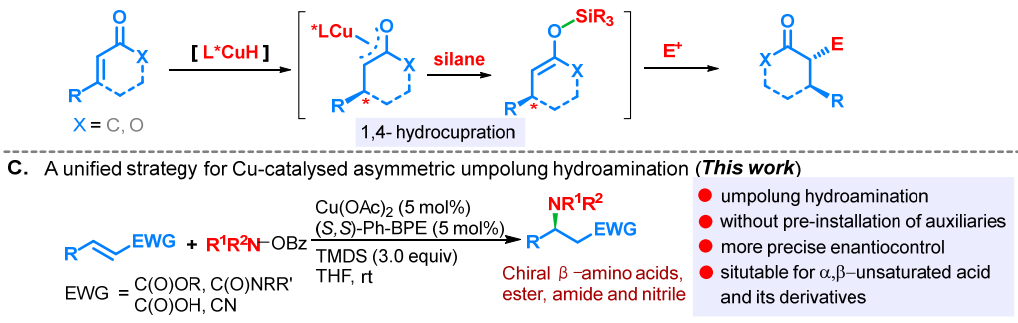

Scheme 1. Approaches for asymmetric aza-Michael addition reaction to $\alpha, \beta$-unsaturated carboxylic acid and its derivatives and CuH-catalyzed transformations of Michael acceptors.

Transition metal-catalyzed asymmetric hydroamination of unsaturated hydrocarbons is a straightforward and powerful approach for rapid assembly of a variety of biologically active chiral amines. ${ }^{10}$ In this content, asymmetric hydroamination of various alkenes and alkynes with in-situ generated $\mathrm{CuH}$ catalysts ${ }^{11,12}$ has attracted much attention since the pioneering reports by Buchwald, Hirano and Miura in 2013. ${ }^{13}$ Besides, an array of electronically-matched CuH-catalyzed asymmetric transformations of Michael acceptors, namely undergoing the 1,4-hydrocupration process, have also been disclosed in the past decades (Scheme 1B)..$^{11 b, 14}$ As our continuing interest in Cu-catalyzed asymmetric hydrofunctionalization of unsaturated hydrocarbons, ${ }^{15}$ herein, we reported a $\mathrm{Cu}$ catalyzed asymmetric umpolung hydroamination of $\alpha, \beta$-unsaturated acid, ester, amide, and nitrile with hydroxylamine derivatives as aminating reagents, providing an unified and straightforward method to synthesize a series of chiral $\beta$-amino acid and its derivatives, without traditional requirement of pre-installation of stoichiometric quantities of auxiliaries. We note that during the preparation of this manuscript, with 1,2-benzisoxazole as the aminating reagent, Guo, Buchwald, and co-worker reported a reversal asymmetric hydroamination of $\beta$-aryl substituted acrylates. In addition, one example of $\alpha, \beta$-unsaturated acid and two examples of $\alpha, \beta$-unsaturated amides were provided. ${ }^{16}$ 
At the outset of our studies, we selected ethyl cinnamate 1a and $O$-benzoylhydroxylamine $4 \mathbf{a}$ as the model substrates in the presence of $5 \mathrm{~mol} \%$ of $\mathrm{Cu}(\mathrm{OAc}) 2$ and $(S)$-DTBM-SEGPHOS L1, the extremely powerful catalytic system of $\mathrm{CuH}$-catalyzed asymmetric hydroamination pioneeringly developed by the group of Buchwald, with an excess of TMDS (1,1,3,3-tetramethyldisiloxane) at room temperature under $\mathrm{N} 2$ atmosphere in THF for $48 \mathrm{~h}$ (Table. 1). In line with the regioselectivity of previous $\mathrm{CuH}$-catalyzed transformations of $\alpha, \beta$-unsaturated ester,[11b,14] the $\alpha$-alkylaminyl substituted product 5a' was obtained in 39\% yield but without enantioinduction. During a thorough evaluation of various chiral ligands, we were surprised to discover that the regioreversed $\beta$ alkylaminyl substituted ester 5a, namely formal aza-Michael addition product, could be obtained and $(S, S)$-Ph-BPE $\mathbf{L} 2$ showed most efficiency in terms of reactivity, regio- and stereocontrol (77\% yield and $97 \%$ ee,). ${ }^{17}$ Moreover, we did not observe the regioisomer $\mathbf{5 a}$ ' with $\mathbf{L 2}$ as the ligand. Examining other commercially available hydrosilanes and solvents were also performed and slightly inferior either the yield or enantiocontrol under the otherwise optimal reaction conditions were observed. ${ }^{17}$

In assessing the scope of the catalytic reaction (Table 1), we found that an array of $\alpha, \beta$-unsaturated esters 1 bearing different steric hindrance functional groups could be efficiently transformed to the corresponding chiral $\beta$-aminyl esters 5a-5e in generally high yields with excellent enantioselectivities. Various substituents including alkyl, methoxyl, phenyl and methylthioyl at either ortho-, meta- or para-position on the aromatic rings of the unsaturated esters 1 were also efficiently converted into chiral products $\mathbf{5 f - 5} \mathbf{k}$ with high level of enantiocontrol. An array of electronic-withdrawing groups, including $-\mathrm{F},-\mathrm{Cl},-\mathrm{Br},-\mathrm{OCF} 3,-\mathrm{CF} 3,-\mathrm{C}(\mathrm{O}) \mathrm{OMe}$, and some privileged heteroaromatic ring motifs widespread in bioactive molecules and pharmaceuticals, such as pyridine and thiophene, were also readily accommodated and provided expected hydroamination products $\mathbf{5 l - 5 u}$ in good yields with excellent enantioselectivities. In addition to examining the scope of $\alpha, \beta$-unsaturated esters, we also surveyed the substrate scope with respect to the hydroxylamine ester component. We found that electron-rich or electron-deficient hydroxylamine esters, nitrogencontaining heterocyclic hydroxylamine esters, as well as the enantiomeric hydroxylamine esters were all applicable to this hydroamination and provided $\beta$-aminyl esters $5 \mathbf{v}-\mathbf{5 z}$ with high efficiency and good to excellent enantiocontrol. Structurally more complicated diaceton-D-glucose and cholesterol-derivated $\alpha, \beta$-unsaturated esters could also be transferred smoothly into corresponding $\beta$-amino ester 5aa and 5ab in good yield with excellent diastereo- and enantiocontrol. The success of this asymmetric hydroamination strategy for $\alpha, \beta$-unsaturated ester encouraged us to continue examining whether this approach could also be applied $\mathrm{t}$ to synthesize chiral $\beta$-aminyl acids and amides. To our delight, without the requirement of further optimizing reaction conditions, a diverse range of $\alpha, \beta$-unsaturated acids with various electronic-withdrawing or donating functional groups on the aromatic rings (2a-2n), including methyl, ethoxyl, ester, phenyl, methylthioyl, halogens, trifluoromethoxyl and trifluoromethyl, and (E)-3-(quinolin-3-yl)acrylic acid (2o) were suitable coupling partners for this amination, providing corresponding $\beta$-alkylaminyl substituted acid 6a-6o with high efficiency and generally excellent level of enantiocontrol. In addition, some representative hydroxylamine esters were also examined and could be readily converted into chiral $\beta$-aminyl acids 6p-6r with over $90 \%$ ee. $\alpha, \beta$-Unsaturated amides 3a-3f could also be converted into corresponding $\beta$-aminyl amides 7a-7f under this catalytic system with high efficiency, despite showing relatively low enantiocontrol compared with that of $\alpha, \beta$-Unsaturated ester and acid. 
Table 1: Scope of hydroamination of $\alpha, \beta$-unsaturated ester, acid and amide.,

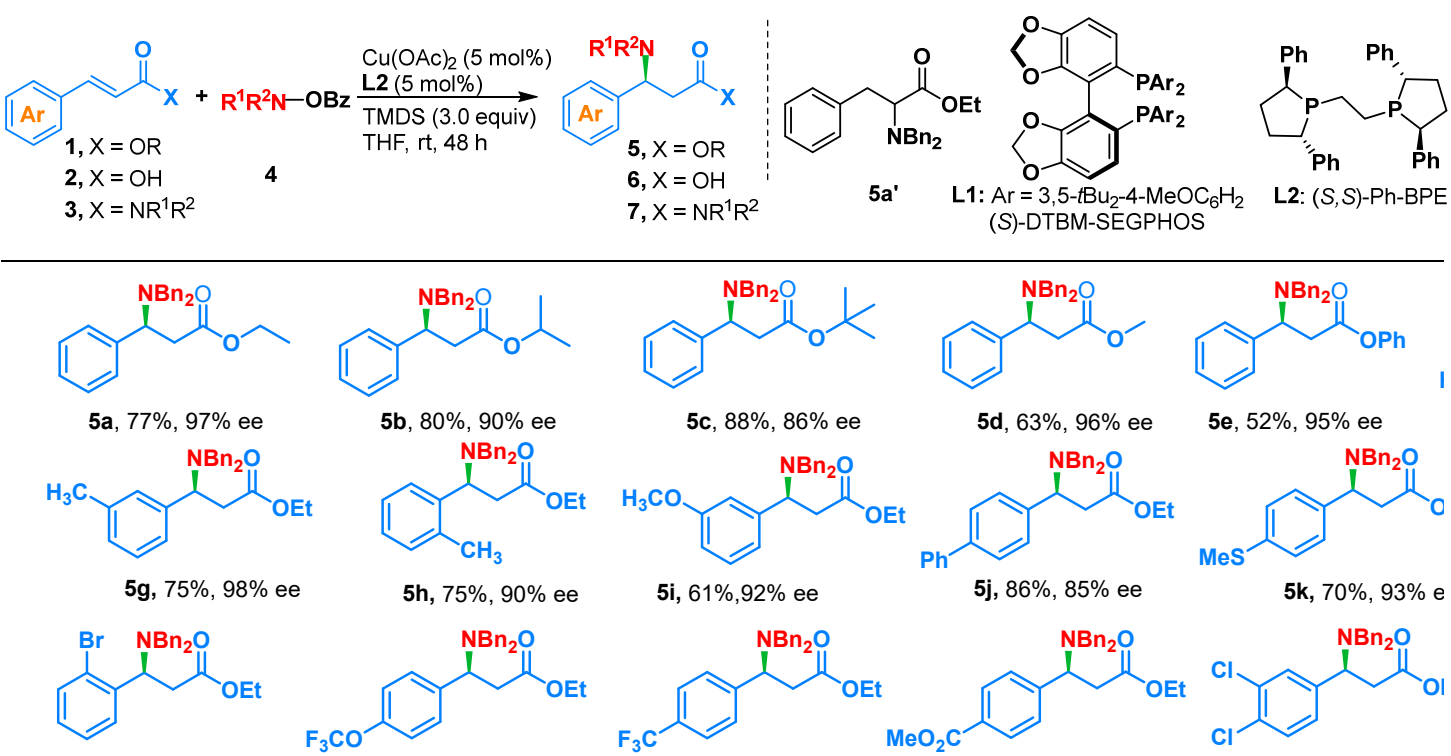

$5 \mathrm{~m}, 74 \%, 90 \%$ ee

5n, $60 \%, 97 \%$ ee

5o, $69 \%, 97 \%$ ee

5p, $60 \%, 97 \%$ ee

$5 q, 68 \%, 79 \%$ ee<smiles>CCOC(=O)C[C@H](N)c1ccc2ccccc2c1</smiles>

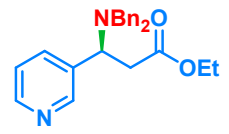<smiles>CCCC(CC(=O)OCC)c1cccs1</smiles>

5s, $69 \%, 84 \%$ ee

5t, $51 \%, 97 \%$ ee

5u, $43 \%, 79 \%$ ee<smiles>CCOC(=O)CC(c1ccccc1)N(Cc1ccccc1)C(C)c1ccccc1</smiles><smiles>CCOC(=O)C[C@@H](c1ccccc1)N(Cc1ccccc1)C(C)c1ccccc1</smiles>

5y, $89 \%, 90 \%$ ee

5z, $85 \%,-90 \%$ ee<smiles>CCOC(=O)CC(c1ccccc1)N1CCOCC1</smiles><smiles>CCOC(=O)CC(c1ccccc1)N1CCSCC1</smiles>

$\mathbf{5 v}, 72 \%, 98 \%$ ee

$5 \mathrm{w}, 82 \%, 92 \%$ ee
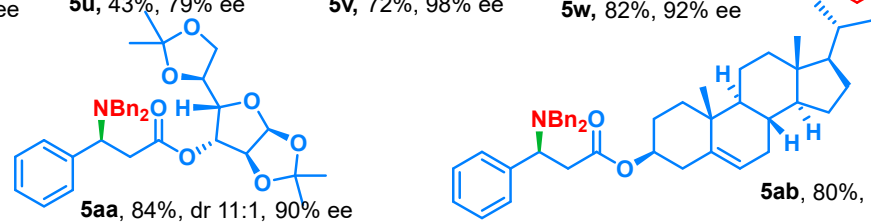<smiles>CCCCC(CC(=O)O)c1ccccc1</smiles><smiles>CCO[C@@H](CC(=O)O)c1ccccc1C</smiles>

$6 b, 92 \%, 90 \% \mathrm{ee}^{[\mathrm{c}]}$

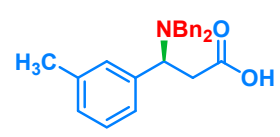

6c, $78 \%, 85 \% \mathrm{ee}^{[\mathrm{c}]}$<smiles>Cc1ccc(C(CC(=O)O)CC(=O)O)cc1</smiles>

$6 d, 85 \%, 89 \%$ ee ${ }^{[c]}$<smiles>CCOc1cccc(C(CC(=O)O)CC(C)(C)C)c1</smiles>

$6 e, 70 \%, 97 \% \mathrm{ee}^{[\mathrm{c}]}$<smiles>CCCCC(CC(=O)O)c1ccc(-c2ccccc2)cc1</smiles>

6 g, $72 \%, 98 \% \mathrm{ee}^{[\mathrm{c}]}$<smiles>CCCCC(CC(=O)O)c1ccc(S(C)(C)C)cc1</smiles>

6h, $78 \%, 83 \% \mathrm{ee}^{[\mathrm{c}]}$<smiles>O=C(O)CC(CC(=O)O)c1cccc(F)c1</smiles>

$6 i, 75 \%, 85 \% e^{[c]}$<smiles>CCCCC(CC(=O)O)c1cccc(Cl)c1</smiles>

6j, $79 \%, 80 \% \mathrm{ee}^{[\mathrm{c}]}$<smiles>CCC(C)[C@H](CC(=O)O)c1ccc(Cl)cc1</smiles>

6 k, $55 \%, 93 \% \mathrm{ee}^{[\mathrm{c}]}$<smiles>CCCCC(CC(=O)O)c1cccc(OC(F)(F)F)c1</smiles>

$6 \mathrm{~m}, 69 \%, 87 \% \mathrm{ee}^{[\mathrm{c}]}$<smiles>CCCC[C@H](CC(=O)O)c1ccccc1C(F)(F)F</smiles>

6 n, $69 \%, 90 \% \mathrm{ee}^{[\mathrm{c}]}$<smiles>CCCCC(CC(=O)O)c1cnc2ccccc2c1</smiles>

6o $51 \%, 90 \% e^{[c]}$<smiles>CCC(C)C(CC(=O)N1CCOC1=O)c1ccccc1</smiles>

7c, $68 \%, 66 \%$ ee<smiles>CC(c1ccccc1)N(Cc1ccccc1)C(CC(=O)O)c1ccccc1</smiles>

6p, $68 \%, 96 \% \mathrm{ee}^{[\mathrm{c}]}$

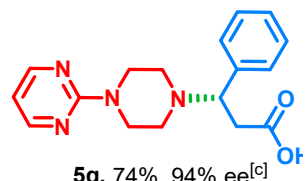

$5 \mathbf{q}, 74 \%, 94 \% \mathrm{ee}^{[\mathrm{c}]}$<smiles>CCC(C)C(CC(=O)N1CCOCC1)c1ccccc1</smiles>

$7 a, 80 \%, 98 \%$ ee<smiles>CCCc1ccc(C(CC(C)([NH3+])c2ccccc2)C(=O)N2CCOCC2)cc1</smiles>

7b, $80 \%, 92 \%$ ee<smiles>CCCCNC(=O)CC(C)c1ccccc1</smiles>

7d, $81 \%, 77 \%$ ee

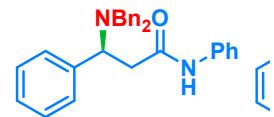

$7 e, 48 \%, 78 \%$ ee

a Reaction conditions: $\alpha, \beta$-unsaturated carbonyl compounds $(0.2 \mathrm{mmol}), 4$ ( $0.3 \mathrm{mmol}, 1.5$ equiv), TMDS (3.0 equiv), $\mathrm{Cu}(\mathrm{OAc})_{2}(5 \mathrm{~mol} \%)$ and chiral ligand $\mathbf{L 2}(5 \mathrm{~mol} \%)$ in $2.0 \mathrm{~mL}$ dry THF at room temperature for $48 \mathrm{~h}$. b Yield determined by ${ }^{1} \mathrm{H}$ NMR spectroscopy using $\mathrm{CH}_{2} \mathrm{Br}_{2}$ as an internal standard and ee value determined by HPLC.c 6.0 equiv TMDS was used and the reactions were performed at $50{ }^{\circ} \mathrm{C}$.

Inspired by the above encouraging results, we next particularly interested that whether this umpolung hydroamination strategy could be applicable to $\alpha, \beta$-unsaturated nitriles to form synthetically formidable challenging chiral $\beta$-amino nitriles. We then began our investigation using 
(E)-cinnamonitrile 8 as the Michael acceptor. To our delight, the expected $\beta$-amino substituted nitrile 9a was indeed generated in $91 \%$ yield and $92 \%$ ee without any further optimization reaction conditions. Encouraged by this result, we then commenced assessment of substrate scope and the functional group compatibility (Table 2). In general, this reaction also showed good functional group tolerance and had nearly negligible electronic effect. For example, either electron-donating functional groups (-CH3, - $\mathrm{OCH} 3,-\mathrm{SCH} 3,-\mathrm{C} 6 \mathrm{H} 5$, - OAc, and -OC6H5) or electron-withdrawing functional groups (-F, $-\mathrm{Cl},-\mathrm{Br}$, $-\mathrm{OCF} 3$, and $-\mathrm{CF} 3)$ on the aromatic rings of the $\alpha, \beta$-unsaturated nitriles, all of which tolerated well and corresponding substrates converted smoothly into desired chiral $\beta$-amino nitriles 9b-9r in good to excellent yields with more than $90 \%$ ee values in most cases. Two representative aminating reagents were also examined and could enable the nitrogencontaining substructures to integrate into the expected chiral products $\mathbf{9 s}$ and $\mathbf{9 t}$ with high efficiency and good enantiocontrol. In addition, the absolute configuration of the product 90 was unequivocally determined by single-crystal X-ray diffraction.

Table 2: Scope of hydroamination of $\alpha, \beta$-unsaturated nitrile. ${ }^{\mathrm{a}, \mathrm{b}}$

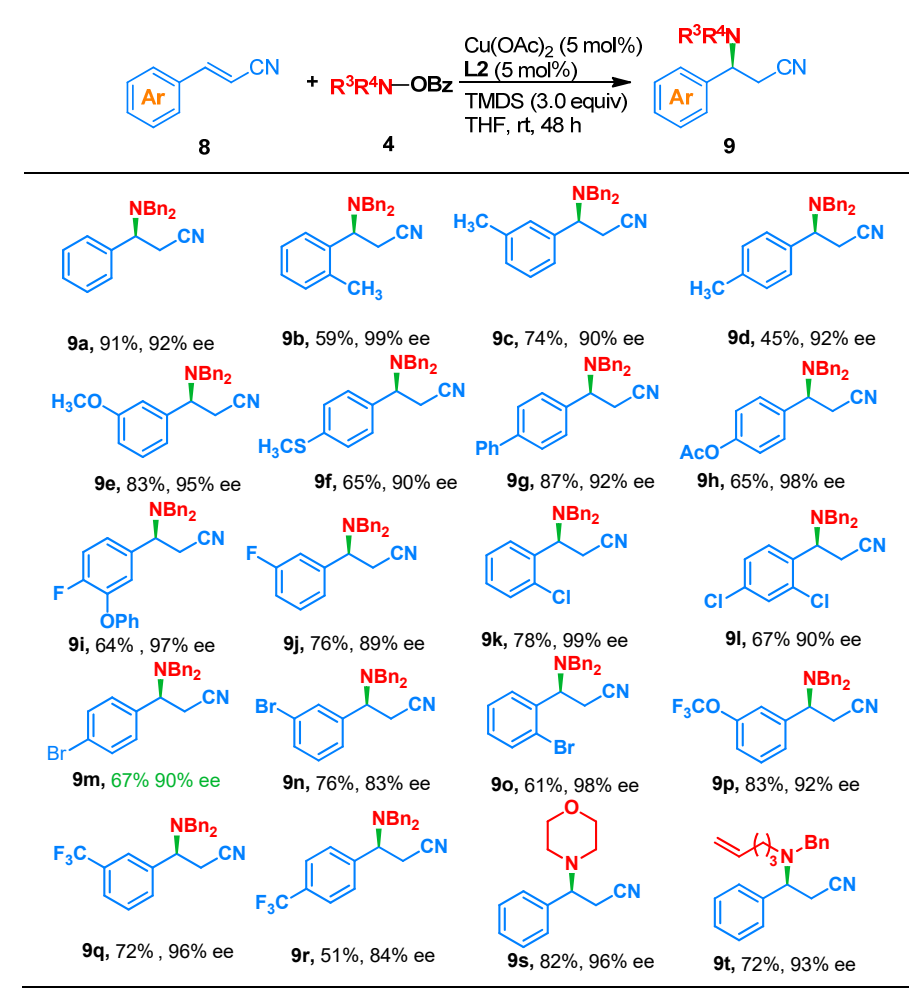

a Reaction conditions: $\alpha, \beta$-unsaturated nitrile $8(0.2 \mathrm{mmol}), 4\left(0.3 \mathrm{mmol}, 1.5\right.$ equiv), TMDS ( 3.0 equiv), $\mathrm{Cu}(\mathrm{OAc})_{2}$ (5 mol\%) and chiral ligand $\mathbf{L 2}(5 \mathrm{~mol} \%)$ in $2.0 \mathrm{~mL}$ dry THF at room temperature for $48 \mathrm{~h}$. b Yield determined by ${ }^{1} \mathrm{H}$ NMR spectroscopy using $\mathrm{CH}_{2} \mathrm{Br}_{2}$ as an internal standard and ee value determined by HPLC.

A gram-scale synthesis was conducted to demonstrate the practicability of this method, the target chiral $\beta$-amino ester $\mathbf{5 a}$ was obtained in good yield without any erosion in enantioselectivity with 1 mol\% $\mathrm{CuCl}$ and $1 \mathrm{~mol} \%$ chiral ligand $\mathbf{L 2}$ (Scheme $2 \mathrm{~A}$ ). We also examined further applications of this chiral compound (Scheme 2B). For instance, chiral $\beta$-amino ester 5a would undergo a selective mono-debenzylation or reduction process, offering chiral $\beta$-amino ester 10 and important chiral $\gamma$ amino alcohol 11 in excellent yield with $95 \%$ and $92 \%$ ee, respectively. In addition, ester 5a can also convert into the chiral cyclic $\beta$-amino ketone $\mathbf{1 2}$ smoothly in $68 \%$ yield with slightly erosion of enantioselectivity. The chiral $\beta$-amino ester $\mathbf{5 a}$ could be further functionalized at the $\alpha$ - 
position of the ester group and deliver more complex molecules. For example, 5a could be efficiently allylated with allyl bromide in the presence of KHMDS, providing synthetically versatile compound $\mathbf{1 3}$ with 2:1 diastereoselectivity. Moreover, without any racemization at the chiral center in 5a was observed under harsh conditions (e.g. in the presence of $\mathrm{NaOH}$ or TFA), that indicate these types of chiral compounds might have the capacity to transfer into more complicated chiral compounds, meanwhile maintating the enantioselectivity.

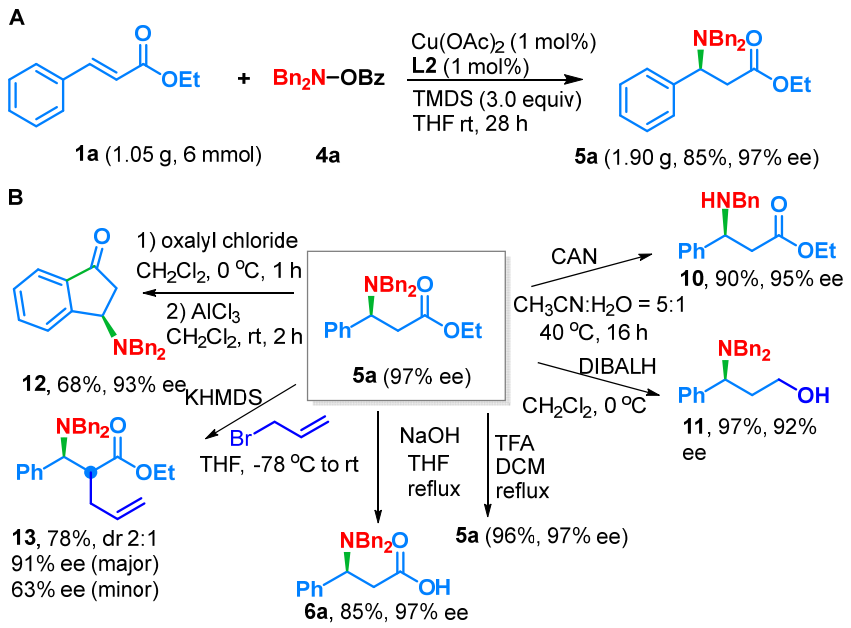

Scheme 2. Gram-scale synthesis and applications of chiral $\beta$-amino ester

In summary, we have developed an efficient umpolung hydroamination strategy of $\alpha, \beta$ unsaturated carboxylic acid, ester, amide, and nitrile through precious choice of chiral ligand, providing an unified approach for the convenient and rapid synthesis of an array of important chiral $\beta$-amino acids and their derivates with high level of regio- and enantiocontrol. Although further studies need to carry out to illuminate the origin of this unusual regio- and eantionselectivity, this approach not only provides an alternative route to traditional aza-Micchael addition, but also opens a new door for the challenging asymmetric addition of other nucleophiles to low reactivity of Michael acceptors. Mechanism studies are currently under way in our lab.

\section{Acknowledgements}

We acknowledge the NSFC (21672033, 21801039, and 21831002), Jilin Educational Committee (JJKH20190269KJ), the Fundamental Research Funds for the Central Universities, and Ten Thousand Talents Program for generous financial support.

Keywords: asymmetric catalysis $\bullet$ umpolung hydroamination $\bullet$ chiral $\beta$-amino acid and its derivatives $\bullet$ copper catalysis

1 a) L-W. Xu, C.-G. Xia, Eur. J. Org. Chem. 2005, 633-639; b) D. Enders, C. Wang, J. X. Liebich, Chem. Eur. J. 2009, 15, 11058-11076; c) M. Sánchez-Roselló, J. L. Aceña, A. Simón-Fuentes, C. del Pozo, Chem. Soc. Rev. 2014, 43, 7430-7453; d) G. Desimoni, G. Faita, P. Quadrelli, Chem. Rev. 2015, 115, 9922-9980; e) J. Wang, P. Li, P. Y. Choy, A. S. C. Chan, F. Y. Kwong, ChemCatChem 2012, 4, 917-925;; f) K. Zheng, X. Liu, $\mathrm{X}$. Feng, Chem. Rev. 2018, 118, 7586-7656.

2 a) Enantioselective Synthesis of $\beta$-Amino Acids. (Ed. Juaristi, E), Wiley-VCH: New York, 1997; b) Z. Amara, J. Caron, D. Joseph, Nat. Prod. Rep. 2013, 30, 1211-1225; c) Highlights in Bioorganic Chemistry; (Eds.: C. Schmuck, H. Wennemers), Wiley-VCH: Weinheim, 2004. d) G. Cardillo, C. Tomasini, Chem. Soc. Rev. 1996, 25, 117-128; e) Cephalosporins and Penicillins Chemistry and Biology; (Ed.: Flynn, E. H), Academic Press: New York and London, 1972.

3 a) Comprehensive Asymmetric Catalysis I-III, (Eds.: E. N. Jacobsen, A. Pfaltz, H. Yamamoto), Springer, Berlin, 1999. b) Catalytic Asymmetric Conjugate Reactions, (Ed.: A. Córdova),Wiley-VCH, 2010. 
4 For reviews, see: a) B. Weiner, W. Szymański, D. B. Janssen, A. J. Minnaard, B. L. Feringa, Chem. Soc. Rev. 2010, 39, 1656-1691; b) D. Monge, H. Jiang, Y. Alvarez-Casao, Chem. Eur. J. 2015, 21, 4494-4504; c) K. M. Byrd, Beilstein J. Org. Chem. 2015, 11, 530-562. For leading examples: see: c) L. Falborg, K. A. Jørgensen, J. Chem.Soc., Perkin Trans. 1 1996, 2823-2826; d) H. Jiang, M. W. Paixão, D. Monge, K. A. Jørgensen, J. Am. Chem. Soc. 2010, 132, 2775-2783; e) M. P. Sibi, J. J. Shay, M. Liu, C. P. Jasperse, J. Am. Chem. Soc. 1998, 120, 6615-6616; f) M. P. Sibi, M. Liu, Org. Lett. 2001, 3, 4181-4184; g) M. P. Sibi, N. Prabagaran, S. G. Ghorpade, C. P. Jasperse, J. Am. Chem. Soc. 2003, 125, 11796-11797; h) J. K. Myers, E. N. Jacobsen, J. Am. Chem. Soc. 1999, 121, 8959-8960; i) M. Gandelman, E. N. Jacoben, Angew. Chem. Int. Ed. 2005, 44, 2393 -2397; Angew. Chem. 2005, 117, $2445-2449 ;$ j) N. Yamagiwa, H. Qin, S. Matsunage, M. Shibasaki, J. Am. Chem. Soc. 2005, 127, 13419-13427; k) S. Matsunage, T. Kinoshita, S. Okada, S. Harada, M. Shibasaki, J. Am. Chem. Soc. 2004, 126, 7559-7570; 1) T. E. Horstmann, D. J. Guerin, S. J. Miller, Angew. Chem. Int. Ed. 2000, 39, 3635-3638; Angew. Chem. 2000, 112, 3781-3784 m) D. J. Guerin, S. J. Miller, J. Am. Chem. Soc. 2002, 124, 2134-2136; n) S. Kikuchi, H. Sato, S. Fukuzawa, Synlett 2006, 7, 1023-1026; o) B. E. Uno, R. D. Dicken, L. R. Redfern, C. M. Stern, G. G. Krzywicki, K. A. Scheidt, Chem. Sci. 2018, 9, 1634-1639

5 N. Hayama, R. Kuramoto, T. Földes, K. Nishibayashi, Y. Kobayashi, I. Pápai, Y. Takemoto, J. Am. Chem. Soc. 2018, 140, 12216-12225;

6 a) M. Bandini, A. Eichholzer, M. Tragni, A. Umani-Ronchi, Angew. Chem. Int. Ed. 2008, 47, 3238 -3241; Angew. Chem. 2008, 120, 3282-3285; b) Y. Lin, W. J. Hirschi, A. Kunadia, A. Paul, I. Ghiviriga, K. A. Abboud, R. W. Karugu, M. J. Vetticatt, J. S. Hirschi, D. Seidel, J. Am. Chem. Soc. 2020, 142, 5627-5635.

7 a) N. J. Weise, F. Parmeggiani, S. T. Ahmed, N. J. Turner, J. Am. Chem. Soc. 2015, 137, 12977-12983; b) R. Li, H. J. Wijma, L. Song, Y. Cui, M. Otzen, Y. Tian, J. Du, T. Li, D. Niu, Y. Chen, J. Feng, J. Han, H. Chen, Y. Tao, D. B. Janssen, B. Wu. Nature Chemical Biology 2018, 14, 664-670....

8 A. J. Fatiadi, In Preparation and Synthetic Applications of Cyano Compounds, (Eds.: S. Patai and Z. Rappaport), Wiley, New York, 1983.

9 a) L. Fadini, A. Togni, Chem. Commun. 2003, 30-31; b) L. Fadini, A. Togni, Helvetica Chimica Acta. 2007, 90, 411-424; c) Z. Yang, D. Liu, Y. Liu, M. Sugiya, T. Imamoto, W. Zhang, Organometallics 2015, 34, $1228-1237$.

10 For selected recent reviews, see: a) T. E. Müller, M. Beller, Chem. Rev. 1998, 98, 675-704; b) F. Alonso, I. P. Beletskaya, M. Yus, Chem. Rev. 2004, 104, 3079-3159; c) T. E. Müller, K. C. Hultzsch, M. Yus, F. Foubelo, M. Tada, Chem. Rev. 2008, 108, 3795-3892; d) L. Huang, M. Arndt, K. Gooßen, H. Heydt, L. Gooßen, Chem. Rev. 2015, 115, 2596-2697; For asymmetric hydroamination of alkenes, see: e) A. L. Reznichenko, A. J. Nawara-Hultzsch, K. C. Hultzsch, Top. Curr. Chem. 2013, 343, 191-260; f) J. Hannedouche, E. Schulz, Chem. Eur. J. 2013, 19, 4972-4985; g) G. Zi, J. Organomet. Chem. 2011, 696, 68-75; h) G. Zi, Dalton Trans. 2009, 9101-9109; i) S. R. Chemler, Org. Biomol. Chem. 2009, 7, 3009-3019; j) I. Aillaud, J. Collin, J. Hannedouche, E. Schulz, Dalton Trans. 2007, 5105-5118; k) K. K. Hii, Pure Appl. Chem. 2006, 78, 341-349.

11 For reviews, see: a) S. Rendler, M. Oestreich, Angew. Chem. Int. Ed. 2007, 46, 498-504; Angew. Chem. 2007, 119, 504-510; b) C. Deutsch, N. Krause, B. H. Lipshutz, Chem. Rev. 2008, 108, 2916-2927; c) T. Fujihara, K. Semba, J. Terao, Y. Tsuji, Catal. Sci. Technol. 2014, 4, $1699-1709$; d) M. T. Pirnot, Y.-M. Wang, S. L. Buchwald, Angew. Chem. Int. Ed. 2016, 55, 48- 57; Angew. Chem. 2016, 128, 48 - 57; e) A. J. Jordan, G. Lalic, J. P. Sadighi, Chem. Rev. 2016, 116, 8318-8372; f) Chen, J., Guo, J., Lu, Z. Chin. J. Chem. 2018, 36, 1075-1109.

12 a) S. Zhu, S. L. Buchwald, J. Am. Chem. Soc. 2014, 136, 15913-15916; b) N. Niljianskul, S. Zhu, S. L. Buchwald, Angew. Chem., Int. Ed. 2015, 54, 1638-1641; Angew. Chem. 2015, 127, 1658-1661; c) S.-L. Shi, S. L. Buchwald, Nat. Chem. 2015, 7, 38-44; d) Y. Miki, K. Hirano, T. Satoh, M. Miura, Org. Lett. 2014, 16, 1498-1501; e) Y Xi, T. W. Butcher, J. Zhang, J. F. Hartwig, Angew. Chem., Int. Ed. 2016, 55, 776-780; f) D. Nishikawa, K. Hirano, M. Miura, J. Am. Chem. Soc. 2015, 137, 15620-15623; g) Y. Yang, S.-L. Shi, D. Niu, P. Liu, S. L. Buchwald, Science 2015, 349, 62-66; h) J. S. Bandar, M. T. Pirnot, S. L. Buchwald, J. Am. Chem. Soc. 2015, 137, 14812-14818; i) S.-L. Shi, Z. L. Wong, S. L. Buchwald, Nature 2016, 532, 353-356; j) S. Tobisch, Chem. Eur. J. 2016, 22, 8290-8300; k) S. Zhu, N. Niljianskul, S. L. Buchwald, Nat. Chem. 2016, 8, 144-150; 1) H. Wang, J. C. Yang, S. L. Buchwald, J. Am. Chem. Soc. 2017, 139, 258428-8431; m) G. Lu, R. Y. Liu, Y. Yang, C. Fang, D. S. Lambrecht, S. L. Buchwald, P. Liu, J. Am. Chem. Soc. 2017, 139, 1654816555; n) Y. Zhou, O. Engl, J. S. Bandar, E. D. Chant, S. L. Buchwald, Angew. Chem., Int. Ed. 2018, 57, 6672-6675; Angew. Chem. 2018, 130, 6782 -6785; o) Q.-F. Xu-Xu, Q.-Q. Liu, X. Zhang, S.-L. You, Angew. Chem. Int. Ed. 2018, 57, 15204 -15208; Angew. Chem. 2018, 130, 15424-15428; p) S. Ichikawa, S. Zhu, S. L. Buchwald, Angew. Chem. Int. Ed. 2018, 57, 8714-8718; Angew. Chem. 2018, 130, 8850-8854; q) S. Guo, J. C. Yang, S. L. Buchwald, J. Am. Chem. Soc. 2018, 140, 15976-15984; r) F. Xie, B. Shen, X. Li, Org. Lett. 2018, 20, 7154-7157; s) A. A. Thomas, K. Speck, I. Kevlishvili, Z. Lu, P. Liu, S. L. Buchwald, J. Am. Chem. Soc. 2018, 140, 13976-13984; t) L. Yu, P. Somfai, Angew. Chem. Int. Ed. 2019, 58, 8551-8555; Angew. Chem. 2019, 131, 8639-8643; u) T. Takata, K. Hirano, M. Miura, Org. Lett. 2019, 21, 4284-4288; v) S. Ichikawa, X.J. Dai, S. L. Buchwald, Org. Lett. 2019, 21, 4370-4373.

13 a) a) Y. Miki, K. Hirano, T. Satoh, M. Miura, Angew. Chem. Int. Ed. 2013, 52, 10830 -10834; Angew. Chem. 2013, 125, 11030-11034; b) S. Zhu, N. Niljianskul, S. L. Buchwald, J. Am. Chem. Soc. 2013, 135, $15746-15749$.

14 a) B. H. Lipshutz, W. Chrisman, K. Noson, P. Papa, J. A. Scalfani, R. W. Vivian, J. M. Keith, Tetrahedron 2000, 56, 2779-2788; b) D. H. Apella, Y. Moritani, R. Shintani, E. M. Ferreira, S. L. Buchwald, J. Am. Chem. Soc. 1999, 121, 9473-9474; c) Y. Moritani, D. H. Apella, V. Jurkauskas, S. L. Buchwald, J. Am. Chem. Soc. 2000, 122, 6797-6798; d) G. Hughes, M. Kimura, S. L.Buchwald, J. Am. Chem. Soc. 2003, 125, 11253-11258; 
e) M. P. Rainka, Y. Aye, S. L. Buchwald, Proc. Natl. Acad. Sci. U.S.A. 2004, 101, 5821-5823; f) B. H. Lipshutz, J. M. Servesko, B. R. Taft, J. Am. Chem. Soc. 2004, 126, 8352-8353.

15 a) G. Xu, H. Zhao, B. Fu, A. Cang, G. Zhang, Q. Zhang, T. Xiong, Q. Zhang, Angew. Chem. Int. Ed. 2017, 56, 13130-13134; Angew. Chemie. 2017, 129, 13310-13314; b) G. Xu, B. Fu, H. Zhao, Y. Li, G. Zhang, Y. Wang, T. Xiong, Q. Zhang, Chem. Sci., 2019, 10, 1802-1806; c) B. Fu, X. Yuan, Y. Li, Y. Wang, Q. Zhang, T. Xiong, Q. Zhang, Org. Lett. 2019, 21, 3576-3580.

16 S. Guo, J. Zhu, S. L. Buchwald, Angew. Chem. Int. Ed. Doi: 10.1002/anie.202007005.

17 See Supplementary Information for details.

G. Zhang,$^{\dagger}$ T. Qin, Prof. Dr. T. Xiong, S. Liu, Prof. Dr. Q. Zhang

Department of Chemistry

Jilin Province Key Laboratory of Organic Functional Molecular Design \& Synthesis

Northeast Normal University, Changchun 130024, China

b Y. Liang, ${ }^{\dagger}$ Prof. Dr. W. Guan

Department of Chemistry

Institute of Functional Material Chemistry

Northeast Normal University, Changchun 130024, China E-mail: guanw580@nenu.edu.cn

c Prof. Dr. Q. Zhang

State Key Laboratory of Organometallic Chemistry

Shanghai Institute of Organic Chemistry, Chinese Academy of Sciences, 345 Lingling Lu, Shanghai 200032, China These authors contributed equally to this work. 